\title{
Molecular neurobiology of Drosophila taste
}

\author{
Erica Gene Freeman ${ }^{1}$ and Anupama Dahanukar ${ }^{1,2}$ \\ ${ }^{1}$ Bioengineering Interdepartmental Graduate Program \\ ${ }^{2}$ Department of Entomology \\ University of California, Riverside, CA 92521, USA
}

Corresponding author: anupama.dahanukar@ucr.edu 


\begin{abstract}
Drosophila is a powerful model in which to study the molecular and cellular basis of taste coding. Flies sense tastants via populations of taste neurons that are activated by compounds of distinct categories. The past few years have borne witness to studies that define the properties of taste neurons, identifying functionally distinct classes of sweet and bitter taste neurons that express unique subsets of gustatory receptor $(G r)$ genes, as well as water, salt, and pheromone sensing neurons that express members of the pickpocket (ppk) or ionotropic receptor (Ir) families. There has also been significant progress in terms of understanding how tastant information is processed and conveyed to higher brain centers, and modulated by prior dietary experience or starvation.
\end{abstract}




\section{Introduction}

Insects rely on their taste system to evaluate the palatability of food sources and make decisions to consume nutritious foods and avoid harmful substances. Taste neurons are organized in hair like structures called sensilla, which are distributed in various organs in the fly body (Figure 1), including the labellum at the distal tip of the proboscis, the distal tarsal segments of the legs, and pharyngeal organs lining the esophagus, all of which regulate feeding behaviors. Taste sensilla are also present on anterior wing margins and the ovipositor, which may be involved in aspects of grooming and egg laying behaviors.

In recent years, members of the gustatory receptor (Gr) family, which was identified several years ago, have been systematically mapped to sweet and bitter taste neurons in various organs [1-4]. More recently members of the pickpocket (ppk) family of epithelial sodium channels [5-7], the transient receptor potential family of cation channels [8-10], and the ionotropic receptor (Ir) family [11,12] have been associated with the taste system as well. These advances have led to comprehensive maps of receptor expression in taste neurons, revealing fundamental principles about the molecular organization of the gustatory system of the fly.

Significant progress has also been made in characterizing response profiles of taste neurons, revealing their specificity, tuning breadth, and behavioral roles [1-3]. These studies have highlighted the complexity of stimulus representation in the taste system as a whole, and have invited experiments to probe the extent to which the fly can use this information for both hard-wired and experience-guided behaviors. 
Functional analyses have also uncovered unexpected interactions between aversive tastants and appetitive neurons and have revealed at least two distinct mechanisms by which sweet taste neuron activity is inhibited by bitter tastants $[13,14]$.

One area in which our understanding of the gustatory system has lagged behind is the identification of higher order neurons in taste circuits and the representation of taste stimuli in higher brain centers. Recent studies that describe the identification of sweet projection neurons [15], and tastant-evoked activation of neurons in the calyx of the mushroom body [16], have begun to address these questions.

\section{Molecular and functional organization of taste neurons}

The adult fly: The morphology and distribution of taste sensilla is remarkably stereotypical between individuals (Figure 1), which has facilitated systematic analyses of gene expression and function of taste neurons housed within each sensillum. Such analyses have yielded detailed receptor-to-neuron maps of the two major external taste organs - the labellum (Figure 1A) [1,4], and the tarsi (Figure 1B) [2] - as well as initial analysis of the labral sense organ in the pharynx (Figure 1C) [3]. In the labellum, each of the $\sim 60$ sensilla contains up to four taste neurons that are molecularly and physiologically distinct from each other, and are selectively activated by tastants that are palatable (sweet, salty, water) or noxious (high salt, bitter, low pH).

Sweet taste neurons express members of a conserved clade of Grs related to the trehalose receptor Gr5a. Reporter gene expression studies suggest some molecular 
diversity between sweet taste neurons in different sensilla, although selected sugars and glycerol activate most sweet taste neurons that have been profiled [1-4]. Recent functional analyses suggest that pharyngeal sweet taste neurons exhibit more selective responses as compared to those in the labellum [3], and reveal at least three classes of sweet taste neurons in tarsi based on the strength and selectivity of their responses to sugars [2]. Testing additional sweet tastants may expose further functional diversity within these neurons, which may correspond to their receptor expression patterns.

Bitter or deterrent taste neurons found in the labellum and tarsi are activated by various compounds that taste bitter to humans. In contrast to the relative homogeneity of sweet taste neurons, bitter taste neurons fall into distinct classes by virtue of their response profiles and their molecular signatures of overlapping but unique subsets of Gr genes [1,2]. Although tastant panels have been limited in size, they nevertheless reveal bitter taste neurons of both broadly and narrowly tuned varieties. Bitter taste neurons in S-type sensilla also respond to high concentrations of salt. Interestingly, functional classes of deterrent neurons in the labellum are distinct from those found in the tarsi, suggesting that bitter tastant space may be sampled differentially by different organs $[1,2]$. Bitter neurons in tarsi and wings also respond to microbial lipopolysaccharides and trigger grooming behaviors [17]. Functional variations between taste organs are supported by the observation that distinct subsets of $\mathrm{Gr}$ genes are expressed in labellar, tarsal and pharyngeal organs [1-3], and also that bitter neurons in different organs can have opposite effects on feeding and oviposition behaviors [18]. 
Gr-labeled sweet and bitter taste neurons also co-express receptors of other families. A systematic expression analysis of Ir20a family [12], which comprise a clade of the ionotropic receptor (Ir) family, shows distinct stereotypical expression patterns in neurons of the labellum, tarsi and pharynx, including Gr-labeled sweet and bitter neurons, as well as those that are not labeled by any Grs [12]. Some bitter neurons also express members of the Transient receptor potential (Trp) family $[9,10]$.

There also exist distinct populations of neurons dedicated for the detection of water (low osmolarity) and salt. A water neuron is found in every labellar sensillum that houses four taste neurons. The salt neuron is housed in L-type labellar sensilla. Both drive appetitive behaviors for water and salt, respectively. Recent studies have uncovered that these neuronal classes do not express Grs, but members of the pickpocket (ppk) and Ir families $[5,6,11]$. Some ppk genes also mark populations of neurons that are involved in detection of pheromones [7], discussed in more detail in an accompanying article.

The larva: The larval taste system is simpler than that of the adult, and yet, organized in a similar manner by which tastants of various categories - sweet, bitter, salt and amino acids - are detected. Larval taste organs are the dorsal, terminal, and ventral organs on the head, and three pharyngeal organs. As in the adult, members of Gr, ppk, and Ir families are expressed in larval taste neurons [19-21], defining sub-populations that are likely to have distinct functional roles. $G r$ and $I r$ genes that are expressed include some that are specific to larvae and others that are also in the adult $[19,20]$. Only a single 
sweet receptor, Gr43a, is expressed in the pharynx and brain [22]. Bitter Grs are expressed in various combinations, presumably promoting differences in response profiles between neurons. ppk genes appear to be involved in salt detection [21], and the functions of $I r$ genes remain to be discovered.

\section{Taste detection by sensory neurons}

Sweet: The mechanism by which various sweet tastants are detected by the clade of eight Grs has been a topic of intense study in recent years. Mutant analyses suggest that each receptor of this clade participates in detection of multiple sugars and moreover, the full extent of the response to each sugar is dependent on more than one of these receptors [4,23]. Recent success with ectopic expression of individual sweet receptors in an olfactory neuron corroborates the idea that each Gr participates directly in ligand recognition [23]. The Grs appear to be broadly divided into two groups depending upon whether their response profiles are similar to Gr5a or Gr64a, and explain how the collective responses of the sweet Grs account for the broad sensitivity of sweet taste neurons. In addition, a conserved receptor outside the sweet clade, Gr43a, also responds to fructose and other sugars. Interestingly, Gr43a is expressed in the brain and is involved in sensing sugar levels in the hemolymph [24]. A recent study suggests that Gr64a may do so as well [4]. Although Grs are molecularly and evolutionarily distinct from the heterodimeric mammalian sweet taste receptor, the occurrence of ligand-binding sites in each subunit appears to be a convergent strategy [25]. 
Nevertheless, several mechanistic details of sweet taste receptor function still need to be worked out. Most vital is that the precise composition of a functional sweet taste receptor in its endogenous context, possibly comprising multiple Gr subunits, remains a mystery. This is in part because the functional expression of sweet Grs in vivo has largely been achieved only in the context of the ab1C olfactory neuron [23], in which Gr21a/Gr63a or other factors may facilitate trafficking, stability or function of sweet Grs. There are open questions about how Grs or Gr complexes transduce signal upon ligand binding. Recent evidence lends support to the idea that Gr proteins display an inverted topology like the related Ors and may function as ligand-gated ion channels [26]. Experimental support for this model comes from studies of a highly conserved receptor outside the sweet clade, Gr43a, which is able to confer fructose sensitivity to excised patches of membranes of Gr43a-expressing cultured cells [27]. However, mutants that disrupt G protein signaling in vivo also cause some defects in sweet taste detection [28-31]. Thus, although direct evidence for metabotropic function is lacking, G protein signaling may be involved in some way.

Fatty acid: A recent study has shown that Drosophila sweet neurons, like those of larger flies, also mediate behavioral responses to fatty acids, which are appetitive at low concentration [32]. Flies in which sweet neurons are genetically silenced have reduced preference for feeding on fatty acids. The function of a phospholipase C, norpA, is required for detection of fatty acids but not for sugars, indicating separable mechanisms for sensing two categories of appetitive stimuli in sweet taste neurons. It therefore 
appears unlikely that fatty acids are detected by one or more of the sweet Grs, but rather by as yet unidentified receptors.

Bitter: Deterrent taste neurons have been grouped into subsets by virtue of their bitter tastant response profiles. Gr-GAL4 analysis suggests that all deterrent neurons express some "core" Grs, which may serve a general function, as well as unique subsets of Grs, which are likely to be important for ligand detection [1]. As predicted, flies lacking one of the core Grs display broad defects in sensing bitter compounds [33-35]. Moreover, overexpression of an I-a class-specific Gr59c in all bitter neurons backs this hypothesis by conferring I-a class ligand sensitivity to berberine, lobeline, and denatonium benzoate in other bitter neurons [1] as well as in the ab1C olfactory neuron [23]. A few other class-specific Grs have been linked to selected compounds using mutant analysis, including Gr93a with caffeine [36] and Gr8a with L-canavanine [37]. As is the case for sweet Grs, the composition and signaling properties of a bitter taste receptor is not yet known. The prevailing idea resting on these observations, and in particular on analysis of $\mathrm{Gr}$ mutants that lose sensitivity to caffeine [36], is that a functional receptor must include at least three Gr subunits. However, this model awaits vetting by heterologous expression studies. What will also be interesting to determine is how co-expression of multiple functional receptors, as is thought to be the case in neurons that express thirty or so Grs, combines to account for the response spectra of deterrent neurons.

Deterrent taste neurons also respond to irritants such as allyl isothiocyanate in wasabi, and acrolein in cigarette smoke. TrpA1, an evolutionarily conserved cation 
channel, acts in GRNs present in the pharynx to prevent ingestion of these compounds [8]. TrpA1 is also co-expressed with Grs in labellar bitter taste neurons, where it is required for detection of aristolochic acid [9]. Multiple signal transduction pathways must operate in bitter taste neurons, because loss of TrpA1 function impacts responses to aristolochic acid but not to other bitter tastants like caffeine, quinine or strychnine [9]. Moreover, TrpA1 functions via independent molecular mechanisms to fulfill its roles in detecting reactive electrophiles and bitter compounds. Heterologous expression of TrpA1 can confer sensitivity to reactive electrophiles but not to aristolochic acid $[8,9]$. Responses to the latter are also dependent on Phospholipase C, suggesting that TrpA1 functions downstream of a PLC signaling cascade for this function. Another Trp channel, Trpl, is also expressed in bitter taste neurons, and mediates responses to camphor [10]. Ectopic expression of Trpl in sweet taste neurons or cultured cells is sufficient for camphor responsiveness. Thus, like TrpA1, Trpl also directly recognizes tastants.

Acid: Electrophysiological analysis of labellar sensilla found that two of the five classes of deterrent neurons are also activated by acidic $\mathrm{pH}$ [38]. Although these neurons are not dedicated "sour" cells as described in mammals, the sub-specialization of a population of bitter taste neurons for detecting acids may nevertheless afford the fly the means to distinguish acidic compounds from other aversive chemicals. Grs that specifically label the class of acid-sensitive deterrent neurons have been reported previously [1]. However, sensitivity to low pH was not altered by loss of Gr33a, a receptor required broadly in deterrent neurons [38]. Investigation of other receptors, 
including those implicated in sensing acids in other organisms, also yielded no candidates. Thus, the acid-activated receptor(s) in these neurons remains to be identified. Given that acid taste receptors have not been found in any animal, the cellular identification of acid-responsive neurons provides a foundation with which to pursue this question.

Salt: The behavioral valence to salt depends on its concentration. Low salt is appetitive, whereas high salt is aversive. Taste neurons that were classically characterized as "salt" neurons in L-type labellar sensilla display peak responses to $\sim 100 \mathrm{mM}$ sodium chloride and evoke appetitive behavior. Ir76b, which marks this population of neurons, is necessary for the attractive response to salt and confers salt sensitivity when expressed in sweet neurons [11]. Mutational analysis of a residue in a transmembrane region that controls ion conductance in related iGluRs suggests that Ir76b may be fixed in a sodium permeable state, but there may be little sodium conductance, until exposure to salt, in the low $\mathrm{Na}^{+}$sensillar lymph that bathes the dendritic membranes of GRNs. Flies lacking Ir76b continue to reject high concentrations of salt and now also reject low concentrations of salt, indicating that salt detection by deterrent neurons is intact and likely occurs via a different receptor. Interestingly, Ir76b also appears to be expressed in gustatory neurons that don't respond to salt, as well as in several classes of olfactory neurons that are presumably salt insensitive [39]. Whether, and if so, how Ir76b channel activity is gated in these neurons remains to be determined. 
Drosophila larvae can also detect and respond to salt across a wide concentration range, with low salt being appetitive and high salt being aversive. Salt taste in larvae appears to be dependent on ppk genes. An RNAi screen identified ppk11 and ppk19 as genes required for behavioral attraction to low salt and salt sensitivity in the terminal organ [21]. Similar to that in the adult, high salt response is genetically separable from low salt response. Behavioral aversion to high salt relies on ppk19 and serrano [40]. The ppk genes may not be necessary for salt taste in the adult fly, raising questions about why there exist two different molecular mechanisms for low salt taste.

Water: ppk28, a member of the DEG/ENaC family of pickpocket receptors in Drosophila, is responsible for sensing pure water or low osmolarity in taste neurons that are dedicated for this purpose. The molecular identification of water taste neurons and the role of ppk28 in sensing water were uncovered by genetic studies, including enhancer trap labeling of these neurons [41], and analysis of ppk28 [5,6] - loss of ppk28 renders these neurons incapable of responding to water, and heterologous expression of ppk28 in bitter taste neurons or in cultured cells confers sensitivity to water. As observed for the water neuron in vivo, heterologously expressed ppk28 is inhibited by a variety of solutes, confirming its role as an osmosensor. Related epithelial sodium channel proteins are involved in sensing salt in mammalian taste buds [42]. How ppk28 differs from sodium sensing ENaCs in terms of ligand recognition and channel activity will be interesting to determine. 
Amino acid: Only recently have behavioral responses to pure amino acids been described in Drosophila, which are enhanced in animals deprived of dietary protein [43]. Although changes in feeding preference could well be attributed to internal sensing mechanisms, the enhancement of proboscis extension in response to stimulation by amino acid solutions supports the existence of peripheral detection in taste neurons. However, amino acid sensing taste neurons or receptors have not yet been reported in Drosophila.

Carbonation: Certain food sources of the fly, such as yeasts and rotting fruit, release carbon dioxide. A population of neurons innervating the oral taste pegs was found to be specifically tuned to carbonated water and did not respond to any other tested stimuli including sugars, salt, amino acids, acids or bitter compounds [44]. Artificial activation of these neurons drives consumption but carbonated water by itself has not been shown to stimulate food intake. While two gustatory receptors, Gr21a and Gr63a, mediate detection of carbon dioxide in olfactory neurons $[45,46]$, these receptors don't appear to be responsible for sensing the taste of carbonation. The molecular mechanism underlying the detection of carbonated water is not yet known.

\section{Taste modulation at the periphery}

Recent studies have uncovered evidence for modulation of sensory neuron activity prior to the first relay. These exciting new findings suggest the presence of multiple molecular and cellular mechanisms by which tastant information is integrated in primary sensory neurons. One general theme that emerges is that aversive tastants, 
including bitter compounds and acids, can inhibit the activity of appetitive taste circuits in both adults and larvae (Figure $2 A)[14,38,47]$. The observed depression in the firing rate of sweet neurons when testing mixtures of sucrose and the aversive tastants is independent of the activity of the deterrent neuron $[14,38,47]$. Bitter tastants, for example, can do so either directly via the action of Obp49a, an odorant binding protein present in the sensillar lymph [13], or indirectly via GABAergic interneurons that connect bitter taste neuron activity to that of sweet taste neurons [48]. Behavioral analyses yield results as predicted, because sugar content being equal, ingestion of acid-laced mixtures decreases as the $\mathrm{pH}$ falls, even in flies in which deterrent taste neurons are genetically ablated [38]. Interestingly, low concentrations of acid tastants have also been observed to modulate detection of bitter compounds in the context of both sweet and deterrent neurons, suppressing their inhibitory effect in the former and their excitatory effect in the latter [49]. Whether the mechanisms by which carboxylic acids or low $\mathrm{pH}$ inhibit taste neurons are similar to those implemented by bitter tastants remains to be determined. Nevertheless, it appears that cell autonomous modulation of sweet neuron activity is a common strategy for regulating ingestion of aversive tastants in the context of mixed food sources.

Other studies have found that taste neuron sensitivity is modulated by prior dietary experience (Figures $2 \mathrm{~B}$ and $2 \mathrm{C}$ ). Response to a non-toxic bitter compound, camphor, was decreased after prolonged exposure of the flies to camphor [10]. The change in sensitivity is brought about by an E3 ubiquitin ligase-regulated decline in the levels of Trpl, which is required for camphor response. Moreover, both Trpl levels and 
behavioral tolerance for camphor were restored once the flies were returned to their standard diet. Elimination of all calories from the diet also caused increased activity in the $\mathrm{Gr} 5 \mathrm{a}^{+}$sweet taste circuit $[15,50]$, and reduced sensitivity in the bitter taste circuit [51]. The former is achieved via dopamine signaling, which may target both primary and secondary neurons in the sweet circuit $[15,50]$. The latter is dependent on SNPF that acts via GABAergic interneurons [51]. The precise mechanisms that alter the sensitivity of primary taste neurons remain to be elucidated.

\section{Taste processing in the brain}

Taste neurons send their input to the central nervous system, where signals from various organs and taste modalities are processed and integrated. Neurons in the labellum and pharynx project to the subesophageal ganglion (SOG), whereas tarsal taste neurons terminate in the SOG as well as in neuromeres of the thoracic ganglia (Figure 3A). Following up on analysis of Gr-GAL4 expression patterns in the periphery, a recent study mapped the axonal projections of neurons labeled by each $G r$ driver in the CNS [52]. The expression of multiple Gr-GAL4 lines in each organ and sensillar structure, as well as the expression of individual Gr-GAL4 lines in multiple locations, both contribute to the overall diversity in projection patterns. Overall, ten categories of patterns were defined in the SOG and nine in the thoracic abdominal ganglia. Each category is a unique combination of discrete patterns elements that define taste neurons in terms of their taste quality, organ location, and in some instances, sensillar type (Figure 3B). Whether, and if so how, the fly exploits this diversity in projection 
patterns to drive various behavioral responses, will likely be a topic of future investigations.

The SOG has long been known as a primary gustatory center, but little is known about where taste information is conveyed from the SOG (Figure 3C). Recently, second order neurons that relay sweet information from the SOG to the antennal and mechanosensory motor center (AMMC) in the deutocerebrum were described [15]. The AMMC, which also receives input from mechanosensory and olfactory neurons, thus appears to be involved in processing multisensory information. The identification of sweet projection neurons came from anatomical criteria using GFP reconstitution across synaptic partners (GRASP) with Gr5a ${ }^{+}$sweet taste neurons, as well as functional criteria using calcium imaging to show sweet taste-evoked activation in the AMMC. Interestingly, functional analysis of these neurons suggests that they faithfully convey labellar sweet taste to the next relay, and do not integrate information about other locations and taste qualities. Other studies have identified interneurons that impinge on taste circuits and feeding behavior routines, including a command neuron that promotes feeding [53], dopaminergic neurons that promote feeding [54], GABAergic neurons that restrain feeding [55], and neurons in the ventral nerve cord that balance feeding and locomotion [56].

Higher order centers to which the AMMC transmits taste information can now be probed. The mushroom bodies (MB) are likely to emerge as direct or indirect targets, since they are key sites for associative learning. A recent study examined taste 
representations in the MB and found that input to the main calyx continues to be segregated according to taste modality and the location that taste information originates from - bitter and sweet stimuli activate distinct areas, and stimuli from different taste organs activate partially overlapping but distinct patterns [16]. Behavioral analyses support the idea that the fly makes organ-specific taste associations. MB neurons also separate water and sweet qualities, and further, nutritive and non-nutritive sugars $[57,58]$. Unraveling taste circuits, therefore, will be important not only for understanding how sensory input is translated to behavioral output, but also how taste associations are formed in reward and aversive learning.

\section{Conclusions}

Recent studies reveal that Grs, Irs, Trp, and ppk receptors underlie detection of various categories of tastants in Drosophila. Yet, much remains unclear about the composition and response properties of taste receptors, and how the activities of multiple receptor proteins including those belonging to different receptor families (e.g. Gr and Ir), coordinate within the neurons that house them. The development of new ectopic expression tools might enable further analysis of Gr proteins, most of which remain to be deorphanized. With the exception of Ir76b, the function of Ir proteins in the taste system is unknown. It will be interesting to determine whether they function in concert with Grs, or whether they do so independently to recognize other classes of tastants. Finally, it is not known if other Trp channels, ppk proteins, or further candidate receptors are involved. 
Despite the recent characterization of interplay between bitter tastants and sweet neurons via two different mechanisms, the action of different classes of aversive tastants on the different appetitive neurons is not known, Moreover, residual behavioral aversion in flies lacking one or both pathways $[13,14,48]$ suggests that additional mechanisms exist to convey bitter tastant information to sweet neurons. One possibility, which can be tested in ectopic expression systems, is that bitter tastants have direct inhibitory effects on sweet receptors. Other key questions that remain have to do with the identity of neural circuits that process and integrate taste information. Recent reports invite exciting new avenues of investigation to determine the higher brain locations that receive taste input from the AMMC, and to trace the circuits by which information is relayed to motor neurons and neurons of the mushroom body to control feeding behavior and associations with appetitive and aversive learning.

\section{Acknowledgements}

We thank Y.-C. Chen and A. Ganguly for comments on the manuscript. Research in A. Dahanukar's laboratory is supported by grants from the Whitehall Foundation (2010-12-42), National Science Foundation (IOS-1149667), and National Institutes of Health (R21DC012408 and R01DC013587).

\section{References and recommended reading}

1. Weiss LA, Dahanukar A, Kwon JY, Banerjee D, Carlson JR: The molecular and cellular basis of bitter taste in Drosophila. Neuron (2011) 69(2):258-272.

2. $\quad$ Ling F, Dahanukar A, Weiss LA, Kwon JY, Carlson JR: The molecular and cellular basis of taste coding in the legs of Drosophila. J Neurosci (2014) 34(21):7148-7164. 
* The authors create a Gr-to-neuron map and determine the responses of tarsal taste sensilla to a panel of compounds. The study also compares taste responses of tarsi with those of the labellum.

3. LeDue EE, Chen YC, Jung AY, Dahanukar A, Gordon MD: Pharyngeal sense organs drive robust sugar consumption in Drosophila. Nat Commun (2015) 6(6667.

* The authors characterize physiological and behavioral roles of pharyngeal sweet taste neurons and identify a subset of Gr genes that are expressed in these neurons. The study demonstrates that mutant flies lacking all external taste hairs are nevertheless capable of selecting sweet substances by virtue of pharyngeal taste neurons.

4. Fuji S, Yavuz A, Slone J, Jagge C, Song X, Amrein H: Drosophila sugar receptors in sweet taste perception, olfaction, and internal nutrient sensing. Curr Biol (2015) 25(5):621-627.

5. Cameron P, Hiroi M, Ngai J, Scott K: The molecular basis for water taste in Drosophila. Nature (2010) 465(7294):91-95.

6. Chen Z, Wang Q, Wang Z: The amiloride-sensitive epithelial Na+ channel PPK28 is essential for Drosophila gustatory water reception. $J$ Neurosci (2010) 30(18):6247-6252.

7. Pikielny CW: Sexy DEG/ENaC channels involved in gustatory detection of fruit fly pheromones. Sci Signal (2012) 5(249):pe48.

8. Kang K, Pulver SR, Panzano VC, Chang EC, Griffith LC, Theobald DL, Garrity PA: Analysis of Drosophila TRPA1 reveals an ancient origin for human chemical nociception. Nature (2010) 464(7288):597-600.

9. Kim SH, Lee Y, Akitake B, Woodward OM, Guggino WB, Montell C: Drosophila TRPA1 channel mediates chemical avoidance in gustatory receptor neurons. Proc Natl Acad Sci U S A (2010) 107(18):8440-8445.

10. Zhang YV, Raghuwanshi RP, Shen WL, Montell C: Food experience-induced taste desensitization modulated by the Drosophila TRPL channel. Nat Neurosci (2013) 16(10):1468-1476.

* TRPL is identified as a receptor that mediates camphor taste in bitter neurons. Flies fed on a camphor-laced diet downregulate the expression of Trpl in an E3 ubiquitin ligase-depenent manner. 
11. Zhang YV, Ni J, Montell C: The molecular basis for attractive salt-taste coding in Drosophila. Science (2013) 340(6138):1334-1338.

** The study identifies Ir76b as a sodium channel that is required in taste neurons for behavioral attraction to low salt. Without Ir76b, low salt concentrations become aversive.

12. Koh TW, He Z, Gorur-Shandilya S, Menuz K, Larter NK, Stewart S, Carlson JR: The Drosophila Ir20a clade of ionotropic receptors are candidate taste and pheromone receptors. Neuron (2014) 83(4):850-865.

* This study describes the mapping of Ir20a genes in adult gustatory neurons and their axonal projections to the CNS using transgenic reporter gene expression.

13. Jeong YT, Shim J, Oh SR, Yoon HI, Kim CH, Moon SJ, Montell C: An odorantbinding protein required for suppression of sweet taste by bitter chemicals. Neuron (2013) 79(4):725-737.

** The authors show that Obp49a is necessary for inhibition of sweet taste neuron activity by bitter tastants, in the absence of input from bitter taste neurons.

14. French AS, Sellier MJ, Moutaz AA, Guigue A, Chabaud MA, Reeb PD, Mitra A, Grau Y, Soustelle L, Marion-Poll F: Dual mechanism for bitter avoidance in Drosophila. J Neurosci (2015) 35(9):3990-4004.

* The authors demonstrate that sweet taste neurons can be inhibited by bitter compounds in a cell autonomous manner.

15. Kain $P$, Dahanukar A: Secondary taste neurons that convey sweet taste and starvation in the Drosophila brain. Neuron (2015) 85(4):819-832.

** The authors identify second order projections neurons that convey sweet taste input from the subesophageal ganglion (SOG) to the antennal mechanosensory motor center $(\mathrm{AMMC})$ in the brain.

16. Kirkhart C, Scott K: Gustatory learning and processing in the Drosophila mushroom bodies. J Neurosci (2015) 35(15):5950-5958.

** The authors map taste representation in the mushroom body and show that attractive and aversive circuits continue to be segregated in the calyx. The study also shows that input from different taste organs can be distinguished in the calyx.

17. Yanagawa A, Guigue AM, Marion-Poll F: Hygienic grooming is induced by contact chemicals in Drosophila melanogaster. Front Behav Neurosci (2014) 8(254. 
18. Joseph RM, Heberlein U: Tissue-specific activation of a single gustatory receptor produces opposing behavioral responses in Drosophila. Genetics (2012) 192(2):521-532.

19. Kwon JY, Dahanukar A, Weiss LA, Carlson JR: Molecular and cellular organization of the taste system in the Drosophila larva. J Neurosci (2011) 31(43):15300-15309.

20. Stewart S, Koh TW, Ghosh AC, Carlson JR: Candidate ionotropic taste receptors in the Drosophila larva. Proc Natl Acad Sci U S A (2015) 112(14):4195-4201.

21. Liu L, Leonard AS, Motto DG, Feller MA, Price MP, Johnson WA, Welsh MJ: Contribution of Drosophila DEG/ENaC genes to salt taste. Neuron (2003) 39(1):133-146.

22. Mishra D, Miyamoto T, Rezenom YH, Broussard A, Yavuz A, Slone J, Russell $\mathrm{DH}$, Amrein $\mathrm{H}$ : The molecular basis of sugar sensing in Drosophila larvae. Curr Biol (2013) 23(15):1466-1471.

23. Freeman EG, Wisotsky Z, Dahanukar A: Detection of sweet tastants by a conserved group of insect gustatory receptors. Proc Natl Acad Sci U S A (2014) 111(4):1598-1603.

** By using an olfactory neuron, the authors demonstrate that each of the eight receptors of the Drosophila sweet clade can detect unique subsets of sweet compounds. Another Drosophila receptor, Gr43a, and its mosquito ortholog, AgGr25, were also tested in this system and found to respond to sugars.

24. Miyamoto $\mathrm{T}$, Slone $\mathrm{J}$, Song $\mathrm{X}$, Amrein $\mathrm{H}$ : A fructose receptor functions as a nutrient sensor in the Drosophila brain. Cell (2012) 151(5):1113-1125.

25. Xu H, Staszewski L, Tang $\mathrm{H}$, Adler E, Zoller M, Li X: Different functional roles of T1R subunits in the heteromeric taste receptors. Proc Natl Acad Sci U S A (2004) 101(39):14258-14263.

26. Zhang HJ, Anderson AR, Trowell SC, Luo AR, Xiang ZH, Xia QY: Topological and functional characterization of an insect gustatory receptor. PLOS ONE (2011) 6(8):e24111. 
27. Sato K, Tanaka K, Touhara K: Sugar-regulated cation channel formed by an insect gustatory receptor. Proc Natl Acad Sci U S A (2011) 108(28):1168011685.

28. Kain P, Badsha F, Hussain SM, Nair A, Hasan G, Rodrigues V: Mutants in phospholipid signaling attenuate the behavioral response of adult Drosophila to trehalose. Chem Senses (2010) 35(8):663-673.

29. Bredendiek N, Hutte J, Steingraber A, Hatt H, Gisselmann G, Neuhaus EM: Go alpha is involved in sugar perception in Drosophila. Chem Senses (2011) 36(1):69-81.

30. Ueno K, Kohatsu S, Clay C, Forte M, Isono K, Kidokoro Y: Gsalpha is involved in sugar perception in Drosophila melanogaster. J Neurosci (2006) 26(23):6143-6152.

31. Ishimoto $\mathrm{H}$, Takahashi $\mathrm{K}$, Ueda $\mathrm{R}$, Tanimura $\mathrm{T}$ : G-protein gamma subunit $\mathbf{1}$ is required for sugar reception in Drosophila. Embo J (2005) 24(18):3259-3265.

32. Masek P, Keene AC: Drosophila fatty acid taste signals through the PLC pathway in sugar-sensing neurons. PLoS Genet (2013) 9(9):e1003710.

33. Moon SJ, Kottgen $\mathrm{M}$, Jiao $\mathrm{Y}, \mathrm{Xu} \mathrm{H}$, Montell $\mathrm{C}$ : A taste receptor required for the caffeine response in vivo. Curr Biol (2006) 16(18):1812-1817.

34. Moon SJ, Lee Y, Jiao Y, Montell C: A Drosophila gustatory receptor essential for aversive taste and inhibiting male-to-male courtship. Curr Biol (2009) 19(19):1623-1627.

35. Lee Y, Kim SH, Montell C: Avoiding DEET through insect gustatory receptors. Neuron (2010) 67(4):555-561.

36. Lee $\mathrm{Y}$, Moon SJ, Montell C: Multiple gustatory receptors required for the caffeine response in Drosophila. Proc Natl Acad Sci U S A (2009) 106(11):4495-4500.

37. Lee Y, Kang MJ, Shim J, Cheong CU, Moon SJ, Montell C: Gustatory receptors required for avoiding the insecticide L-canavanine. J Neurosci (2012) 32(4):1429-1435. 
38. Charlu S, Wisotsky Z, Medina A, Dahanukar A: Acid sensing by sweet and bitter taste neurons in Drosophila melanogaster. Nat Commun (2013) $4(2042$.

39. Benton R, Vannice KS, Gomez-Diaz C, Vosshall LB: Variant ionotropic glutamate receptors as chemosensory receptors in Drosophila. Cell (2009) 136(1):149-162.

40. Alves G, Salle J, Chaudy S, Dupas S, Maniere G: High-NaCl perception in Drosophila melanogaster. J Neurosci (2014) 34(33):10884-10891.

41. Inoshita T, Tanimura T: Cellular identification of water gustatory receptor neurons and their central projection pattern in Drosophila. Proc Natl Acad Sci U S A (2006) 103(4):1094-1099.

42. Chandrashekar J, Kuhn C, Oka Y, Yarmolinsky DA, Hummler E, Ryba NJ, Zuker CS: The cells and peripheral representation of sodium taste in mice. Nature (2010) 464(7286):297-301.

43. Toshima N, Tanimura $\mathrm{T}$ : Taste preference for amino acids is dependent on internal nutritional state in Drosophila melanogaster. J Exp Biol (2012) 215(Pt 16):2827-2832.

44. Fischler W, Kong P, Marella S, Scott K: The detection of carbonation by the Drosophila gustatory system. Nature (2007) 448(7157):1054-1057.

45. Jones WD, Cayirlioglu P, Kadow IG, Vosshall LB: Two chemosensory receptors together mediate carbon dioxide detection in Drosophila. Nature (2007) 445(7123):86-90.

46. Kwon JY, Dahanukar A, Weiss LA, Carlson JR: The molecular basis of $\mathrm{CO}_{2}$ reception in Drosophila. Proc Natl Acad Sci U S A (2007) 104(9):3574-3578.

47. Konig C, Schleyer M, Leibiger J, El-Keredy A, Gerber B: Bitter-sweet processing in larval Drosophila. Chem Senses (2014) 39(6):489-505.

48. Chu B, Chui V, Mann K, Gordon MD: Presynaptic gain control drives sweet and bitter taste integration in Drosophila. Curr Biol (2014) 24(17):1978-1984. 
* This study shows that GABAergic interneurons mediate inhibition of sweet taste neurons when bitter taste neurons are activated.

49. Chen $\mathrm{Y}$, Amrein $\mathrm{H}$ : Enhancing perception of contaminated food through acid-mediated modulation of taste neuron responses. Curr Biol (2014) 24(17):1969-1977.

50. Inagaki HK, Ben-Tabou de-Leon S, Wong AM, Jagadish S, Ishimoto H, Barnea $\mathrm{G}$, Kitamoto $\mathrm{T}$, Axel $\mathrm{R}$, Anderson DJ: Visualizing neuromodulation in vivo: TANGO-mapping of dopamine signaling reveals appetite control of sugar sensing. Cell (2012) 148(3):583-595.

51. Inagaki HK, Panse KM, Anderson DJ: Independent, reciprocal neuromodulatory control of sweet and bitter taste sensitivity during starvation in Drosophila. Neuron (2014) 84(4):806-820.

52. Kwon JY, Dahanukar A, Weiss LA, Carlson JR: A map of taste neuron projections in the Drosophila CNS. J Biosci (2014) 39(4):565-574.

* The study describes axonal projection patterns of $G r$-labeled taste neurons, identifying 10 categories of patterns in the SOG and 9 categories in the thoracic-abdominal ganglia. Patterns are composites of discrete elements that represent the organ location and sensillum structure that the taste neurons originate from, as well as the taste quality that they detect.

53. Flood TF, Iguchi S, Gorczyca M, White B, Ito K, Yoshihara M: A single pair of interneurons commands the Drosophila feeding motor program. Nature (2013) 499(7456):83-87.

54. Marella S, Mann K, Scott K: Dopaminergic modulation of sucrose acceptance behavior in Drosophila. Neuron (2012) 73(5):941-950.

55. Pool AH, Kvello P, Mann K, Cheung SK, Gordon MD, Wang L, Scott K: Four GABAergic interneurons impose feeding restraint in Drosophila. Neuron (2014) 83(1):164-177.

56. Mann K, Gordon MD, Scott K: A pair of interneurons influences the choice between feeding and locomotion in Drosophila. Neuron (2013) 79(4):754765. 
57. Huetteroth W, Perisse E, Lin S, Klappenbach M, Burke C, Waddell S: Sweet taste and nutrient value subdivide rewarding dopaminergic neurons in Drosophila. Curr Biol (2015) 25(6):751-758.

58. Lin S, Owald D, Chandra V, Talbot C, Huetteroth W, Waddell S: Neural correlates of water reward in thirsty Drosophila. Nat Neurosci (2014) 17(11):1536-1542.

59. Dahanukar A, Lei YT, Kwon JY, Carlson JR: Two Gr genes underlie sugar reception in Drosophila. Neuron (2007) 56(3):503-516.

60. Jiao Y, Moon SJ, Montell C: A Drosophila gustatory receptor required for the responses to sucrose, glucose, and maltose identified by mRNA tagging. Proc Natl Acad Sci U S A (2007) 104(35):14110-14115.

\section{Figure legends}

\section{Figure 1. Receptor-to-neuron maps of taste organs.}

(A) Schematic of sensillar classes in the labellum, defined by receptor expression patterns and functional analysis (left). Tables indicating identified neurons (center) or sensilla (right) with their receptor expression patterns. Maps created from expression studies along with, in some instances, functional studies; with the exception of Gr64a, which is mapped to labellar sweet neurons by functional studies $[59,60]$. Receptors marked with an asterisk are not expressed in every sensillum of the indicated class. Sweet receptors in italics have been mapped by knock-in reporter analysis but not by transgenic reporter experiments. Receptors in bold are broadly expressed in bitter neurons. Expression of $p p k 23$ and ppk29 has been assigned to $\mathrm{S}$ sensilla based on observed pairing of $p p k 23^{+}$cells with Gr66a+ cells. (B) Schematic of sensilla in the female fore tarsi (left) and tables indicating identified neurons (center) or sensilla (right) 
with their receptor expression patterns. (C) Schematic indicating the location of oral taste pegs and the labral sense organ (LSO; chemosensory sensilla in lilac, mechanosensory sensilla in black) and ventral cibarial sense organ (VCSO) in the pharynx. Tables indicating identified neurons (right) with their receptor expression patterns in the LSO and VCSO (center) and the taste pegs (right).

\section{Figure 2. Peripheral modulation of taste input.}

(A) Sweet taste neuron response is directly inhibited by bitter tastants via Obp49a and via GABAergic interneurons upon activation of bitter taste neurons. (B) TRPLdependent taste response to camphor is altered by dietary exposure to camphor via E3 ubiquitin ligase-mediated regulation of TRPL levels. (C) Sweet taste circuit sensitivity is enhanced upon starvation via dopamine signaling; bitter taste circuit sensitivity is depressed upon starvation via sNPF signaling.

\section{Figure 3. Elements of taste circuits in the adult fly.}

(A) Schematic of the first relay of taste circuits indicating projections of taste neurons from the proboscis to the subesophageal ganglion (SOG) and those from tarsi to the thoracic ganglia (TG) and the SOG. Locations of the antennal mechanosensory motor center (AMMC) and the mushroom bodies (MB) are also indicated. (B) Schematic of the SOG and the thoracic abdominal ganglia showing pattern elements of axonal projections of taste neurons. (C) Schematic of the brain indicating the locations of primary (SOG) and secondary (AMMC) taste relays, and the mushroom bodies to which taste input is conveyed. 
A

Labellum

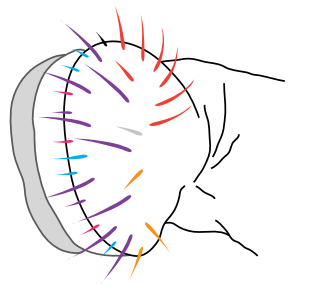

- L

- S-a

- S-b

- I-a

- I-b

Sweet

Bitter

Salt (appetitive)

Water

Deterrent

(uncharacterized)

Uncharacterized

Pheromone

\begin{tabular}{|l|llllll|}
\hline Sensillum & L & S-a & S-b & S-c & I-a & I-b \\
\hline Neurons & & & & & &
\end{tabular}

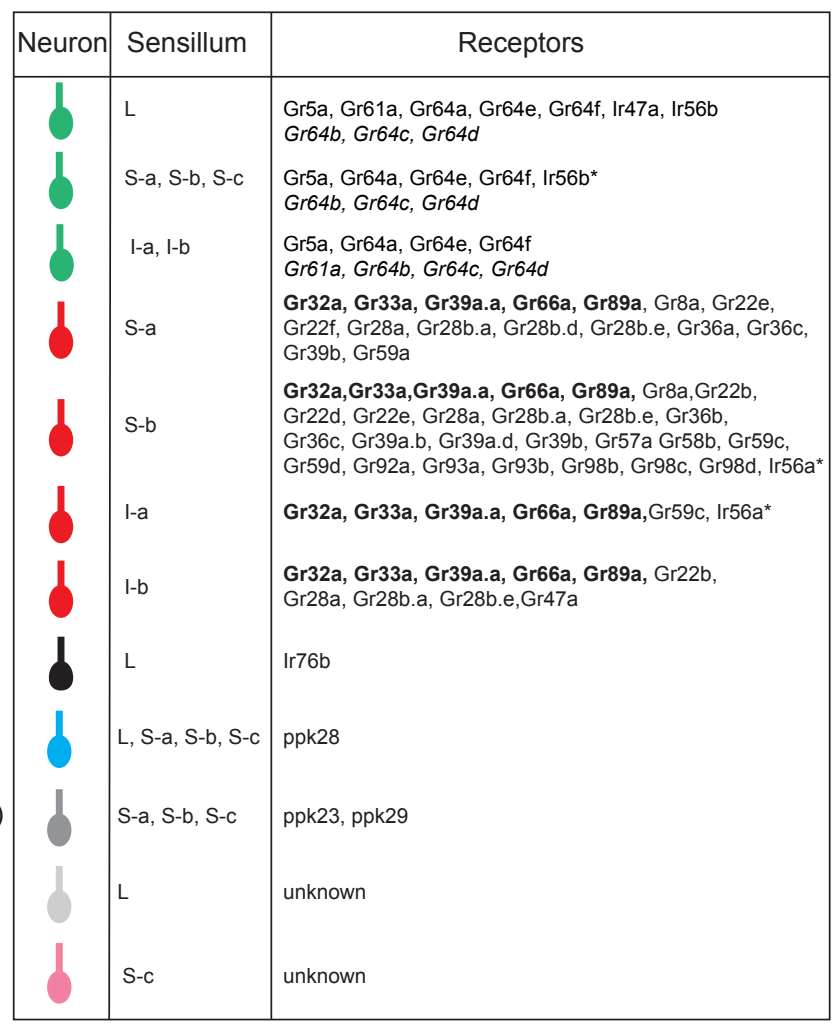

B

\begin{tabular}{|c|c|c|c|}
\hline Temale Tarsi & Neuron & Sensillum & Receptors \\
\hline $\int_{\mathrm{f} 5 \mathrm{v}}^{\mathrm{f}}$ & & $\begin{array}{l}f 2 b, f 3 b \\
f 5 v \\
f 4 b, f 5 s \\
f 5 a \\
f 4 s, f 5 b \\
f 5 s\end{array}$ & $\begin{array}{l}\text { Gr5a, Gr61a, Gr64c, Gr64e, Gr64f } \\
\text { Gr43a, Gr61a, Gr64a, Gr64c, Gr64e, Gr64f } \\
\text { Gr5a, Gr61a, Gr64a, Gr64b, Gr64c, Gr64e, Gr64f } \\
\text { Gr61a, Gr64b, Gr64f } \\
\text { Gr5a, Gr61a, Gr64a, Gr64b, Gr64c, Gr64f } \\
\text { Gr32a, Gr33a, Gr39a,a, Gr89a, Gr58c } \\
\text { Gr33a, Gr39a,a, Gr66a, Gr89a, Gr8a, Gr22b, Gr22a, } \\
\text { Gr22d, Gr28a, Gr28b.c, Gr28b.d, Gr28b.e, Gr39b, } \\
\text { Gr59a, Gr59d, Gr93b, Gr98d }\end{array}$ \\
\hline
\end{tabular}

C

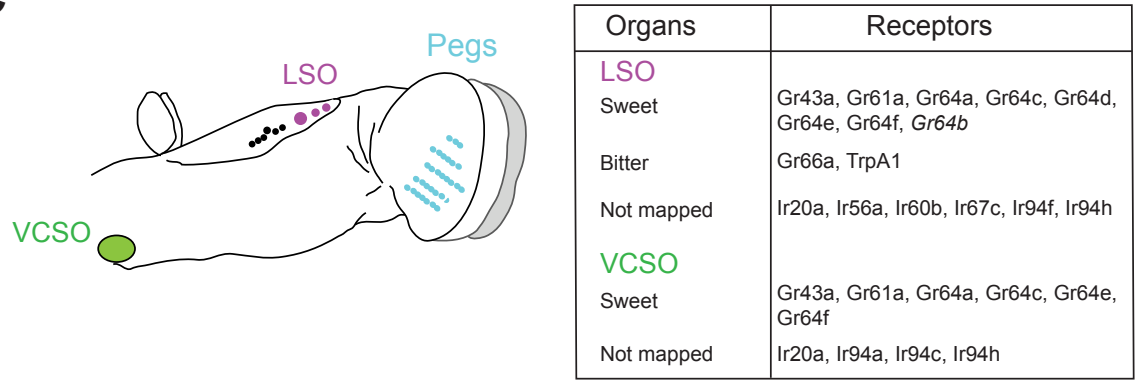

\begin{tabular}{|c|c|}
\hline Sensillum & Receptors \\
\hline I (Sweet, ?) & $\begin{array}{l}\text { Ir56d, Ir94e } \\
\text { (Only in I0) }\end{array}$ \\
\hline $\begin{array}{l}\mathrm{L} \\
\text { (Sweet, ?) }\end{array}$ & $\begin{array}{l}\text { Ir56d, Ir94e } \\
\text { (Only in a subset) }\end{array}$ \\
\hline $\begin{array}{l}\text { S } \\
\text { (Sweet, ?) }\end{array}$ & $\begin{array}{l}\text { Ir47a, Ir56 } \mathrm{d}^{*} \\
\text { (Only in S2 and S6) }\end{array}$ \\
\hline $\begin{array}{l}\mathrm{S} \\
\text { (Deterrent) }\end{array}$ & TrpA1 \\
\hline $\begin{array}{l}\mathrm{S} \\
\text { (Deterrent) }\end{array}$ & TrpL \\
\hline
\end{tabular}

\begin{tabular}{|l|l|}
\hline \multicolumn{1}{|c|}{ Sensillum } & \multicolumn{1}{|c|}{ Receptors } \\
\hline f1a, f1c, f1d, f2a & Ir52a, Ir52c, Ir52d, Ir56d \\
f1b, f3a, f4b, f5a & Ir52a, Ir52c, Ir52d \\
f2b & Ir52a, Ir56b, Ir56d \\
f3b & Ir52a, Ir56b \\
f4s, f5b & Ir47a, Ir56b \\
f5s, f5v \\
$\begin{array}{l}\text { Not mapped } \\
\text { (Low salt) } \\
\begin{array}{l}\text { Not mapped } \\
\text { (Water) }\end{array}\end{array}$ & Ir20a, Ir47a, Ir56b, Ir62a, Ir94h \\
\hline
\end{tabular}

Pegs

\begin{tabular}{|l|l|}
\hline \multicolumn{1}{|c|}{ Taste } & \multicolumn{1}{c|}{ Receptors } \\
\hline Bitter & Gr66a \\
Sweet & Gr5a, Gr64a, Gr64b, \\
& Gr64c, Gr64e, Gr64f \\
Carbonation & unknown \\
Not mapped & Ir56d, Ir76b \\
\hline
\end{tabular}

\section{Figure 1}


A

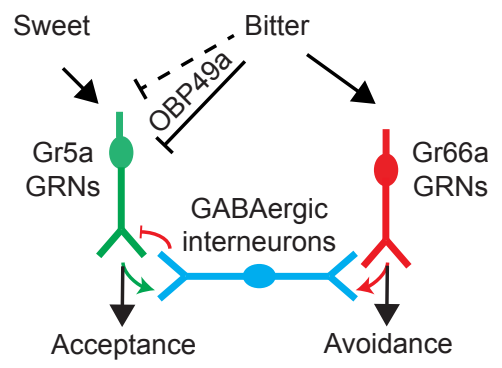

B

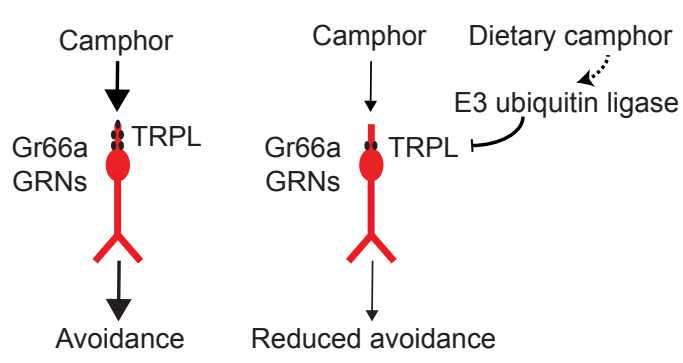

C
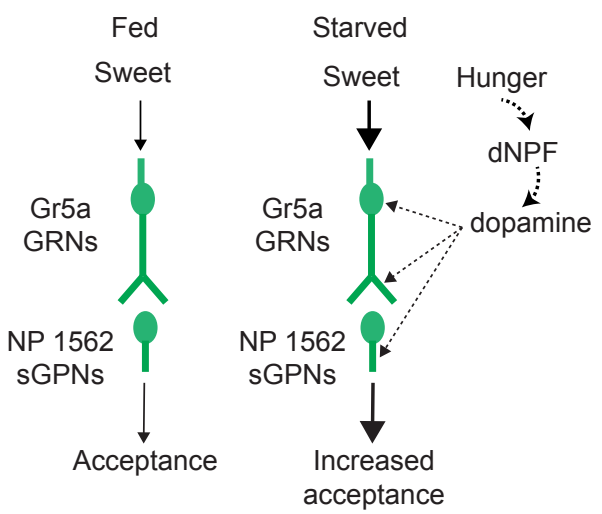

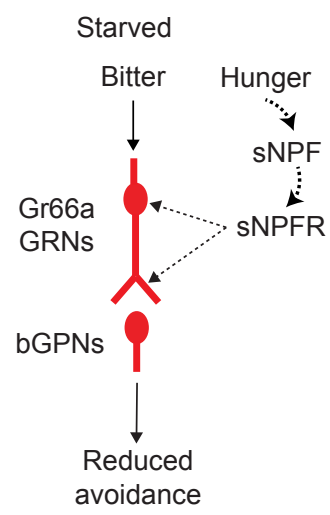

\section{Figure 2}


A

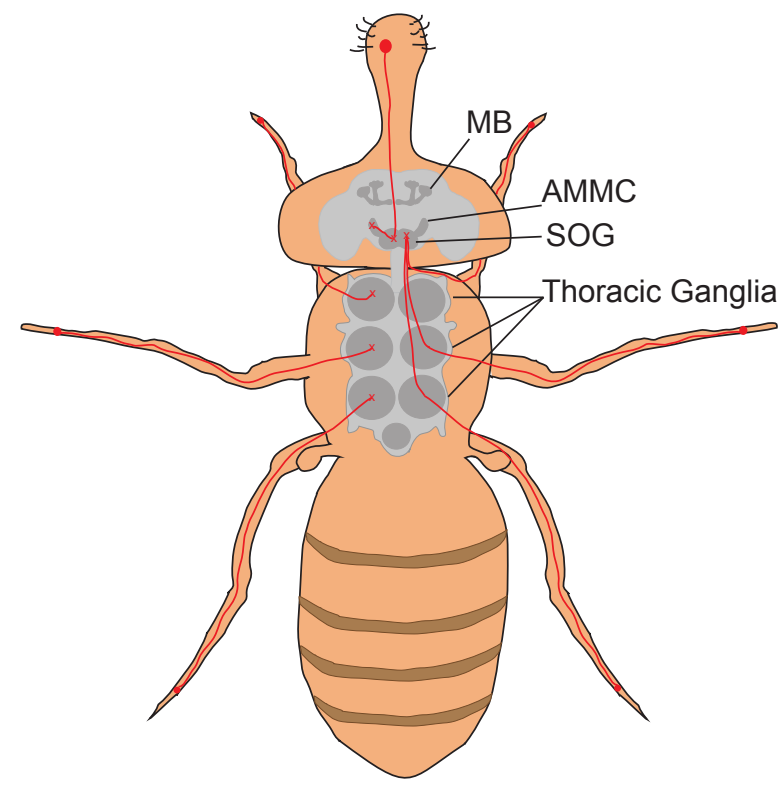

B

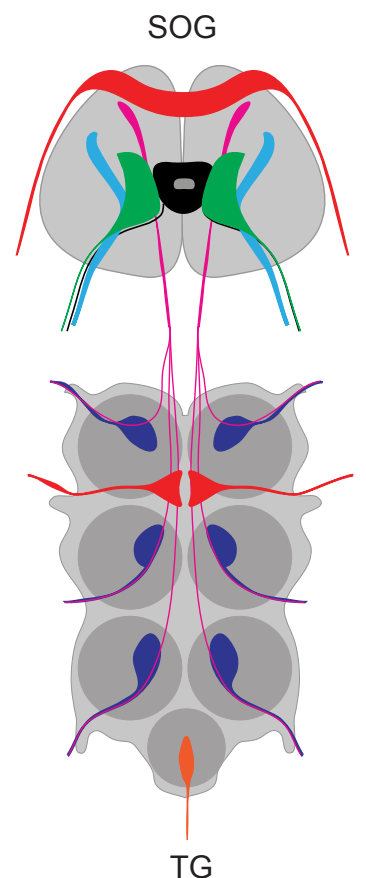

C

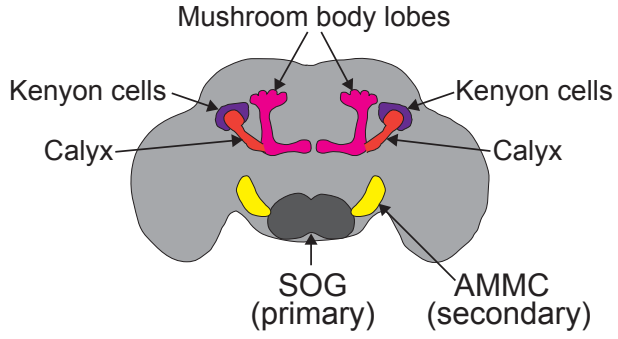

Figure 3 